\title{
Evaluation of cardiovascular toxicity of carbon nanotubes functionalized with sodium hyaluronate in oral regenerative medicine
}

\author{
J.V. Joviano-Santos ${ }^{1}$, M.A. Sá ${ }^{1}$, M.L.A. De Maria ${ }^{1}$, T.C.S. Almeida ${ }^{1}$, V. Geraldo ${ }^{2}$, S. Oliveira ${ }^{2}$, \\ L.O. Ladeira ${ }^{2}$ and A.J. Ferreira ${ }^{1}$ \\ ${ }^{1}$ Departamento de Morfologia, Universidade Federal de Minas Gerais, Belo Horizonte, MG, Brasil \\ ${ }^{2}$ Departamento de Física, Universidade Federal de Minas Gerais, Belo Horizonte, MG, Brasil
}

\begin{abstract}
It has been demonstrated that carbon nanotubes (CNTs) associated with sodium hyaluronate (HY-CNTs) accelerate bone repair in the tooth sockets of rats. Before clinical application of HY-CNTs, it is important to assess their biocompatibility. Moreover, cardiac toxicity may be caused by the translocation of these particles to the blood stream. The aim of this study was to evaluate possible changes in cardiovascular function in male Wistar rats whose tooth sockets were treated with either CNTs or HY-CNTs $(100 \mu \mathrm{g} / \mathrm{mL}, 0.1 \mathrm{~mL})$. Blood pressure and heart rate were monitored in conscious rats 7 days after treatment. Cardiac function was evaluated using the Langendorff perfusion technique. The data showed no changes in blood pressure or heart rate in rats treated with either CNTs or HY-CNTs, and no significant changes in cardiac function were found in any of the groups. To confirm these findings, experiments were conducted in rats injected intraperitoneally with a high concentration of either CNTs or HY-CNTs $(0.75 \mathrm{mg} / \mathrm{kg})$. The same parameters were analyzed and similar results were observed. The results obtained 7 days following injection indicate that the administration of low concentrations of CNTs or HY-CNTs directly into tooth sockets did not cause any significant change in cardiovascular function in the rats. The present findings support the possibility of using these biocomposites in humans.
\end{abstract}

Key words: Cardiac function; Blood pressure; Carbon nanotubes; Nanotechnology; Bone repair; Tooth sockets

\section{Introduction}

Sodium hyaluronate $(\mathrm{HY})$ is one of the main components of the mammalian extracellular matrix and plays an important role in tissue repair (1). HY acts in the wound healing process as a promoter of early inflammation through the production of proinflammatory cytokines and the enhancement of cellular infiltration (2). Regarding bone repair, HY retains osteoinductive growth factors within the local environment due to its chemical properties (3), mediates the adhesion of osteoclasts to the bone surface (4), and accelerates both revascularization (5) and bone formation (5). These features, in addition to its viscoelastic properties and biocompatibility, make HY a suitable candidate for biomedical applications (6). Indeed, by accelerating bone matrix deposition, HY-based gels are potentially useful in the repair process of bone tissue (7). However, the low degree of stability of HY formulations in aqueous environments has hindered their use in oral regenerative medicine.

To increase HY stability, a combination of carbon nanotubes (CNTs) and HY has been proposed as a novel biocomposite, and is referred to as CNTs functionalized with HY (HY-CNTs) $(8,9)$. HY-CNTs have been reported to accelerate the bone repair process in rats following an interval of 7 days after extraction of the upper first molar, when applied to the tooth sockets (9). They have also been shown to enhance bone repair in the tooth sockets of diabetic rats, suggesting usefulness in therapies for bone reconstruction in adverse metabolic states (10). CNTs are allotropic forms of carbon in which a hexagonal matrix is arranged along cylindrical surfaces with diameters on the order of nanometers and lengths on the order of micrometers. Interestingly, the structure of

Correspondence: A.J. Ferreira, Departamento de Morfologia, Universidade Federal de Minas Gerais, Av. Antônio Carlos, 6627, 31270-901 Belo Horizonte, MG, Brasil. Fax: +55-31- 3409-2810. E-mail: anderson@icb.ufmg.br 
carbon atoms gives this nanomaterial a porous threedimensional plane that can act as a scaffold for the nucleation of hydroxyapatite crystals and the growth of cells, such as osteoblasts, which are essential to the bone repair process (11).

To validate the therapeutic potential of CNTs, it is necessary to investigate the possible toxicological effects. A large number of studies stress the importance of knowledge of the cytotoxicity of nanoparticles, especially CNTs, which depends on the experimental model employed, the characteristics of the CNTs (including functionalization), and the parameters tested (12). Thus, the toxicity must be assessed individually for each type of CNT-based biocomposite in every physiological system involved (13). The translocation of these particles to the blood system may cause cardiac toxicity with consequent effects on cardiovascular functions. It has been reported that ultrafine particles can exit the systemic circulation, causing direct cardiovascular effects including cardiac and endothelial injury (14). Thus, the aim of the present study was to evaluate possible cardiovascular changes in rats whose tooth sockets were treated with either CNTs or HY-CNTs.

\section{Material and Methods}

\section{Synthesis, purification, and characterization of single-wall carbon nanotubes}

Single-wall CNTs were synthesized using the electric arc discharge method in an atmosphere of helium at a pressure of 40 Torr. The catalyst was graphite powder doped with nickel, $\mathrm{Y}_{2} \mathrm{O}_{3}$, and FeS at molar ratios of 4.2, 1.0 , and $1.0 \%$, respectively, relative to carbon. CNTs were generated along with impurities, such as amorphous carbon, fullerenes $\mathrm{C} 60$ and $\mathrm{C} 70$, and metallic particles, which were removed in a subsequent purification process. Initially, ultrasound was used to disperse the synthesis product in toluene, and the suspension was then filtered through a 1- $\mu \mathrm{m}$ pore polytetrafluoroethylene (PTFE, Millipore, USA) membrane for the removal of C60 and C70 fullerenes. The deposited material was collected, dried in an oven, subjected to oxidation in air at $390^{\circ} \mathrm{C}$ for $50 \mathrm{~min}$ to remove amorphous carbon, and dispersed in a $6 \mathrm{M} \mathrm{HCl}$ solution for $24 \mathrm{~h}$ to remove the metallic particles. After this acid treatment, the material was filtered through a 1- $\mu \mathrm{m}$ PTFE membrane and oven dried. This step was repeated 3 times to ensure the effective removal of amorphous carbon and metal impurities. The material was then dispersed in deionized water, filtered through a 1- $\mu \mathrm{m}$ PTFE membrane until the $\mathrm{pH}$ of the water reached the range of 6.5 to 7.0 , and then dried in an oven at $90^{\circ} \mathrm{C}$ for $24 \mathrm{~h}$. The characterization of the CNTs using transmission electron microscopy (TEM), scanning electron microscopy (SEM), thermogravimetric analysis, and Raman spectroscopy, and the process of CNT functionalization with $\mathrm{HY}$ are described in detail in previous studies $(9,10)$.

\section{Animals}

Experiments were performed using male Wistar rats weighing 200 to $250 \mathrm{~g}$. The animals were housed in a room with controlled humidity and temperature and a 12:12-h light-dark cycle, with free access to food and water. All procedures were approved by the Ethics Committee on Animal Experimentation of the Universidade Federal de Minas Gerais, Brazil.

\section{Surgical tooth extraction and socket treatments}

The animals were anesthetized with a mixture of $10 \%$ ketamine (Syntec, Brazil) and 2\% xylazine (Vetbrands, Brazil; $1: 1,0.1 \mathrm{~mL} / 100 \mathrm{~g}$ body weight, im) and then placed in the supine position for the extraction of the right and left upper first molars (9). The animals were randomly divided into the following groups: $i$ ) animals with both sockets filled with clot (control), ii) animals with both sockets treated with $\sim 0.1 \mathrm{~mL} 100 \mu \mathrm{g} / \mathrm{mL}$ CNTs (CNT), and iii) animals with both sockets treated with $\sim 0.1 \mathrm{~mL}$ $100 \mu \mathrm{g} / \mathrm{mL}$ HY-CNTs (HY-CNT). The biocomposites were dispersed on carbopol gel and injected into the tooth sockets immediately after extraction using a small, 26gauge needle. It has been reported that carbopol does not interfere with the healing of tooth sockets (9). To maintain the biocomposites at the extraction sites and to avoid hypothermia caused by anesthesia, the rats were kept anesthetized in the supine position on a thermal bed for approximately $4 \mathrm{~h}$. In addition, the animals received pasty chow for 2 days after surgery. The experiments were performed 7 days after tooth extraction. This period, as well as the dose of CNTs and HY-CNTs used, were chosen because this time frame and dose have been found to be able to accelerate the bone repair process in rats $(9)$.

\section{Evaluation of blood pressure and heart rate}

Monitoring of systemic blood pressure and in vivo heart rate $(\mathrm{HR})$ was performed 7 days following tooth extraction. Animals ( $n=5$ per group) were anesthetized with a mixture of $10 \%$ ketamine (Syntec) and $2 \%$ xylazine (Vetbrands; $1: 1,0.1 \mathrm{~mL} / 100 \mathrm{~g}$ body weight, $\mathrm{im}$ ) for the implantation of polyethylene cannulas in the femoral artery. The anesthetized animals were placed in the supine position and, after asepsis with iodine alcohol, an incision was made in the right inguinal region for visualization of the femoral vascular-nervous bundle. A cannula was inserted into the femoral artery and tunneled subcutaneously to the back of the neck. The cannula was filled with saline $(0.9 \% \mathrm{NaCl})$ and the free end was occluded with a metal pin. These procedures were performed $24 \mathrm{~h}$ before recording blood pressure and HR. After the preliminary calibration of the equipment, the cannula was connected to a pressure transducer, and mean arterial pressure (MAP) and HR were recorded for 30 min using a data acquisition system (MP100, Biopac System Inc., USA). Readings were performed, after 
20 min of stabilization, on conscious animals kept in individual boxes.

\section{Analysis of cardiac function}

A Langendorff system with constant pressure (65 $\mathrm{mmHg})$ and oxygenation $\left(5 \% \mathrm{CO}_{2}\right.$ and $\left.95 \% \mathrm{O}_{2}\right)$ was used to evaluate cardiac function in isolated hearts. Animals ( $\mathrm{n}=8-9$ per group) were heparinized (400 IU, ip) $10 \mathrm{~min}$ prior to death by decapitation. The thorax was opened and the heart was dissected, removed, and placed in cold Krebs-Ringer solution (118.4 mM NaCl, $4.7 \mathrm{mM} \mathrm{KCl}$, $1.2 \mathrm{mM} \mathrm{KH} \mathrm{KHO}_{4}, 1.2 \mathrm{mM} \mathrm{MgSO} \mathrm{PH}_{4} .7 \mathrm{H}_{2} \mathrm{O}, 2.5 \mathrm{mM}$ $\mathrm{CaCl}_{2} .2 \mathrm{H}_{2} \mathrm{O}, 11.7 \mathrm{mM} \mathrm{C}_{6} \mathrm{H}_{12} \mathrm{O}_{6}$, and $26.5 \mathrm{mM}$ $\mathrm{NaHCO}_{3}$ ). The ascending aorta was sectioned at its first branch and mounted in the perfusion system. The hearts were perfused through the aortic stump with Krebs-Ringer solution at $37 \pm 1^{\circ} \mathrm{C}$. A small hook was attached to the apex of the left ventricle and connected to a force transducer to record contractile force. Tension of $1.0 \pm 0.2 \mathrm{~g}$ was applied to the heart (diastolic tension) and kept constant during the experiment. HR and the rates of contraction and relaxation $( \pm \mathrm{dT} / \mathrm{dt})$ calculated from the tension readings were stored using a system for the acquisition and analysis of analog-to-digital data (MP100 AcqKnowledge, Biopac Systems Inc.). Coronary flow was measured directly from the effluent for $1 \mathrm{~min}$ every $5 \mathrm{~min}$. The duration of the experiments was $30 \mathrm{~min}$ for the stabilization period followed by $30 \mathrm{~min}$ of recording.

\section{Intraperitoneal protocol}

To confirm the data obtained when CNTs were injected into tooth sockets and to exacerbate any potential toxicological effects of CNTs, an experimental protocol was designed in which the biocomposites were administered intraperitoneally and functional parameters (blood pressure and heart function) were assessed after $24 \mathrm{~h}$. This period was chosen because it seems that $24 \mathrm{~h}$ is the critical period for development of the toxicological effects of nanomaterials (15). In this protocol, a higher dose of CNTs and HY-CNTs was used $(0.75 \mathrm{mg} / \mathrm{kg}$, administered in approximately $3.75 \mathrm{~mL}$ saline). Animals were randomly divided into the following groups: i) control (saline), ii) treatment with CNTs, and iii) treatment with HY-CNTs.

Blood pressure and HR were analyzed before, and 1 day after, the injection of the biocomposites using the tail cuff method ( $n=5$ to 8 animals per group). Briefly, the rats were placed in an acrylic cylindrical tube, which was heated and ventilated. The tails were fitted in a cuff connected to a sphygmomanometer programmed to inflate and deflate automatically at fixed intervals of approximately $50 \mathrm{~s}$. After an acclimation period, the cuff was attached to a transducer that captured blood pressure, pulse, and HR. In this protocol, the animals served as their own controls as MAP and HR were taken before and $24 \mathrm{~h}$ after treatment.

\section{Statistical analysis}

Data are reported as means \pm SE and were analyzed using one-way ANOVA followed by the Newman-Keuls post-test. Tail cuff data (before and 1 day after injection of the biocomposite) were analyzed using the paired Student $t$-test. All analyses were performed using the GraphPad Prism 5 (USA) program. The significance level was set at $5 \%(P<0.05)$.

\section{Results}

TEM analysis showed that the CNTs had few defects in the walls (Figure 1A and B). SEM analysis showed that the purification process was efficient for the removal of impurities from the material (Figure $1 \mathrm{C}$ and $\mathrm{D}$ ). Moreover, the results of thermogravimetric evaluation of as-grown and purified CNTs demonstrated the efficiency of the purification process. In Figure $1 \mathrm{E}$, the dotted line represents the loss of mass of unpurified CNTs in the range of $370^{\circ}$ to $560^{\circ} \mathrm{C}$, which is related to the burning of amorphous carbon and low-quality CNTs, and a $25 \%$ residual mass in the range of $890-1000^{\circ} \mathrm{C}$, which is related to metal oxide impurities. The continuous line is a thermogravimetric curve of purified CNTs, showing a high rate of mass loss at $660^{\circ} \mathrm{C}$ related to the burning of highquality CNTs and an $11 \%$ residual mass of metal carbides or encapsulated metal carbon particles. Thus, the results demonstrate that the CNT purification process was effective in the removal of amorphous carbon and metal nanoparticles, achieving $89 \%$ purity. The purity of the CNTs was further evaluated using Raman spectroscopy (Figure $1 \mathrm{~F}$ and $\mathrm{G}$ ). The frequency of the radial breathing mode was inversely proportional to the diameter of the nanotube. Raman spectra of the material showed a radial breathing mode at $180 \mathrm{~cm}^{-1}$ due to the radial vibration of CNTs with a diameter of $1.4 \mathrm{~nm}$. CNTs with a high degree of structural disorder have a band denominated the $D$ band at $1380 \mathrm{~cm}^{-1}$ and the ratio of the intensity of the $D$ band and $\mathrm{G}$ band is a quantitative characterization of the degree of nanotube perfection. The quality factor $(Q=$ $1-I_{D} / I_{G}$, in which $I_{D}$ is the intensity of the $D$ band and $I_{G}$ is the intensity of $G$ band) was 0.95 and 0.91 for as-grown and purified samples, respectively, which means that the purification process did not generate a significant number of defects.

As depicted in Figure 2A, the MAP of animals whose tooth sockets were treated with CNTs (whether or not functionalized with $\mathrm{HY}$ ) did not differ significantly from that in the control animals. Moreover, no significant differences in the in vivo HR were found among the groups (Figure 2B). Regarding cardiac function, the following parameters were evaluated in isolated heart preparations: systolic tension (Figure 2C), diastolic tension (Figure 2D), $\pm \mathrm{dT} / \mathrm{dt}$ (Figure 2E and F), coronary flow (Figure 2G), and $\mathrm{HR}$ (Figure $2 \mathrm{H}$ ). The injection of either CNTs or HY-CNTs into the tooth sockets did not cause any significant 

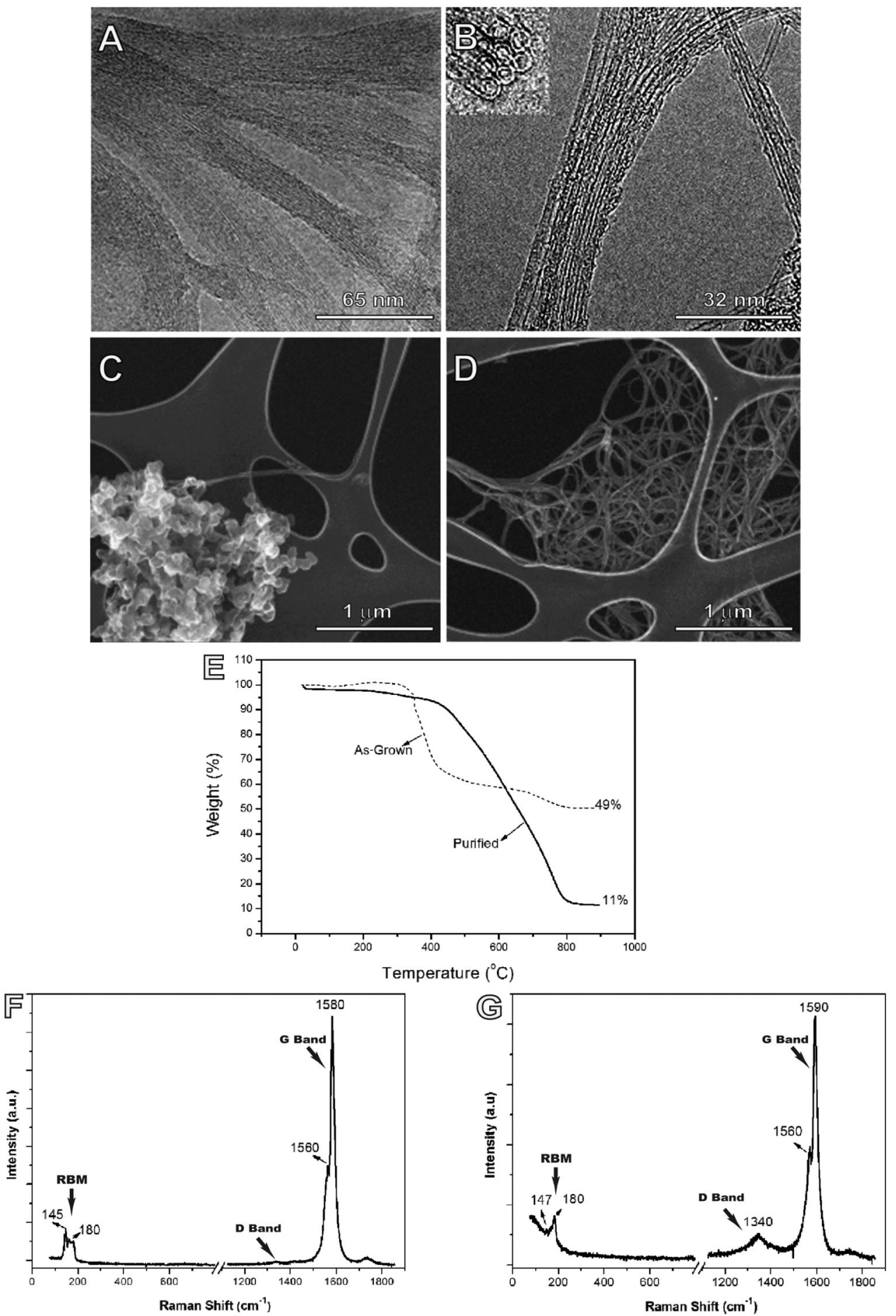

Figure 1. High-resolution transmission electron microscopies ( $A$ and $B$ ) show morphology and few defects in walls of single-wall carbon nanotubes (CNTs) following purification process. Insert in $B$ represents CNTs viewed transversally. $C$ and $D$, Scanning electron microscopy of CNT samples before $(C)$ and after $(D)$ the purification process. $E$, Thermogravimetric analysis of as-grown and purified CNTs. Dotted line represents mass loss of unpurified (as-grown) CNTs and continuous line corresponds to purified CNTs. Raman spectroscopy of CNTs showing low-frequency mode at $180 \mathrm{~cm}^{-1}$, D band at $1340 \mathrm{~cm}^{-1}$, and G band at $1580 \mathrm{~cm}^{-1}$ of unpurified (as-grown) CNTs $(F)$ and purified CNTs $(G)$. RBM: radial breathing mode; a.u.: arbitrary units. 

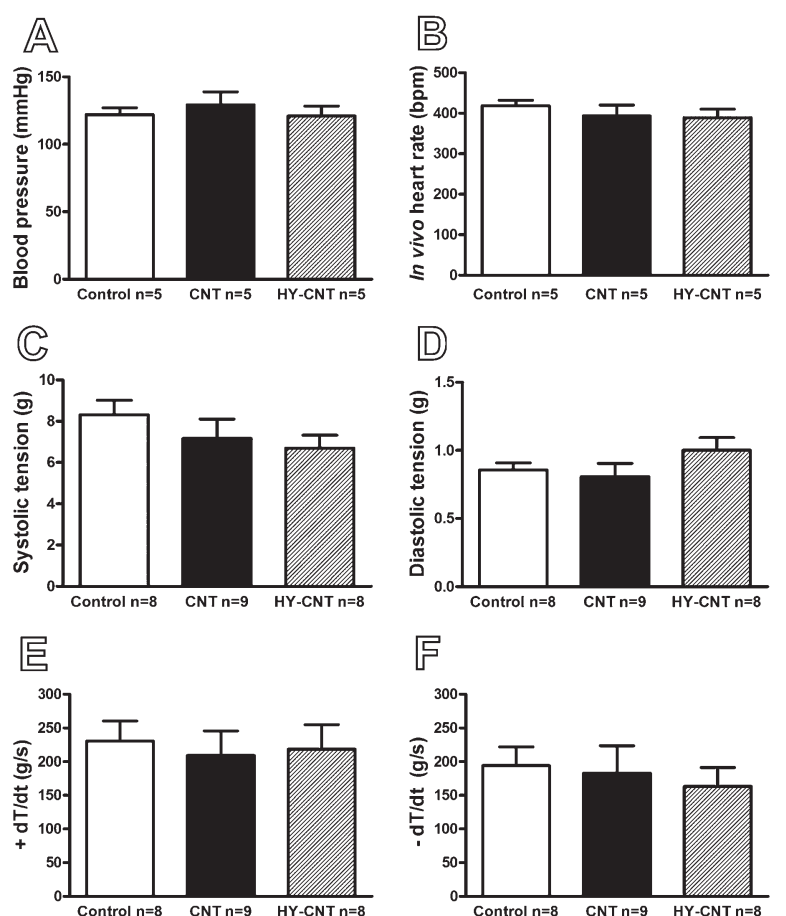

尼

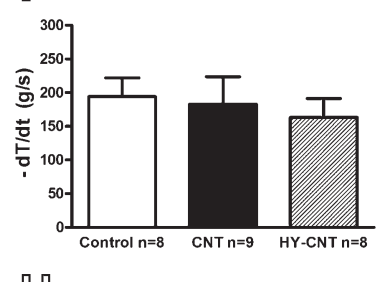

(G)

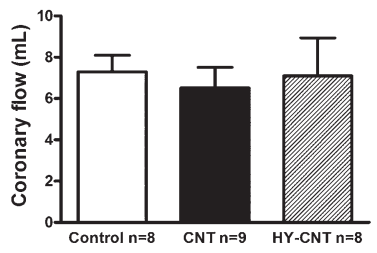

近

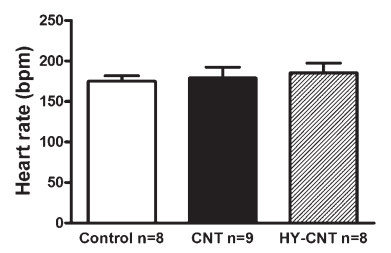

Figure 2. Effects of carbon nanotubes (CNTs) and CNTs associated with sodium hyaluronate (HY-CNTs) on mean arterial pressure (MAP), heart rate (HR) and cardiac function administered to tooth sockets. $A$, MAP; $B$, in vivo $\mathrm{HR} ; C$, systolic tension; $D$, diastolic tension; $E,+\mathrm{dT} / \mathrm{dt} ; F,-\mathrm{dT} / \mathrm{dt} ; G$, coronary flow; $H$, ex vivo $\mathrm{HR} ; \pm \mathrm{dT} / \mathrm{dt}$ : rates of contraction and relaxation. Data are reported as means $\pm \mathrm{SE}$. There were no significant differences among groups $(P>0.05$; one-way ANOVA followed by NewmanKeuls post-test).

changes in these parameters compared with the control group.

The biocomposites were injected intraperitoneally at a high concentration to exacerbate any potential cardiovascular damage caused by either the CNTs or HY-CNTs. Despite the increased bioavailability of these materials, no significant changes were found in MAP (control, $110 \pm$ $6 \mathrm{mmHg}$ before and $100 \pm 4 \mathrm{mmHg} 1$ day after injection; CNTs, $113 \pm 4 \mathrm{mmHg}$ before and $108 \pm 3 \mathrm{mmHg} 1$ day after injection; HY-CNTs, $114 \pm 3 \mathrm{mmHg}$ before and $114 \pm$ $6 \mathrm{mmHg} 1$ day after injection). Moreover, these treatments did not alter HR compared with baseline (control, $376 \pm 14$ bpm before and $373 \pm 13$ bpm 1 day after injection;
CNTs, $369 \pm 9 \mathrm{bpm}$ before and $377 \pm 20$ bpm 1 day after injection; HY-CNTs, $345 \pm 22 \mathrm{bpm}$ before and $374 \pm 16 \mathrm{bpm}$ 1 day after injection). The analysis of cardiac function (systolic and diastolic tension, $\pm \mathrm{dT} / \mathrm{dt}$, coronary flow, and $\mathrm{HR}$ ) also revealed no significant differences among the groups (Table 1).

\section{Discussion}

In the present study, CNTs and HY-CNTs used as biomaterials to accelerate bone repair in the tooth sockets of rats had no significant toxicological effects on cardiovascular function (blood pressure, HR, and cardiac function). Moreover, a high concentration of these biocomposites was administered intraperitoneally to exacerbate any potential harmful effect; but, once again, no significant effects on the cardiovascular system were found.

Due to the importance of CNTs in different applications, especially medicine, it has become imperative to understand the toxicity and in vivo behavior of these materials. Conflicting results regarding clearance, distribution, and toxicity are reported in the literature $(14,16)$, which is likely due to the characteristics of the material used, i.e., differences in the physical properties and structure, as well as pretreatment processes, surfaces, aggregation, purity, functionalization, experimental protocol, administration form, and concentration. Thus, the toxicity must be assessed individually for each type of CNT-based biocomposite and in each physiological system involved. If CNTs and HY-CNTs are to be employed in clinical applications for bone repair, it is crucial to establish the safety of these biocomposites under such conditions. Based on the present experimental protocol, i.e., a low concentration of CNTs/HY-CNTs $(100 \mu \mathrm{g} / \mathrm{mL})$ administered directly into tooth sockets and evaluated 7 days after injection, it is tempting to conclude that this therapeutic approach was safe. The 7day period was chosen because this time frame has been found to be sufficient to accelerate the bone repair process in rats (9).

In general, the present results are in keeping with previous reports showing that CNTs have low toxicity, low immunogenicity, and good biocompatibility (16). For example, a previous study found no apparent changes in cell viability of cardiomyocytes, even when a higher dose of CNTs was used (17). In the present study, no significant changes in cardiac function were found. Moreover, CNTs are compatible with other mammalian cell types, i.e., neurons (16).

In addition to increasing the dispersibility of CNTs in water by modifying their hydrophobic nature (18), it has been proposed that the functionalization process reduces the toxicity of these materials (19). Indeed, this may have contributed to the present findings, since the CNTs used in this study presented carboxylic acid groups bound to 
Table 1. Cardiac function of rats administered intraperitoneally with CNTs or HY-CNTs.

\begin{tabular}{lccc}
\hline & Control $(\mathrm{n}=5)$ & CNTs $(\mathrm{n}=7)$ & HY-CNTs $(\mathrm{n}=5)$ \\
\hline Systolic tension $(\mathrm{g})$ & $9.1 \pm 1.5$ & $8.0 \pm 1.6$ & $10.0 \pm 2.0$ \\
Diastolic tension $(\mathrm{g})$ & $0.9 \pm 0.1$ & $0.9 \pm 0.03$ & $1.0 \pm 0.02$ \\
$+\mathrm{dT} / \mathrm{dt}(\mathrm{g} / \mathrm{s})$ & $252.2 \pm 53.0$ & $183.6 \pm 44.1$ & $304.8 \pm 57.1$ \\
$-\mathrm{dT} / \mathrm{dt}(\mathrm{g} / \mathrm{s})$ & $199.5 \pm 49.8$ & $120.9 \pm 20.3$ & $188.6 \pm 44.3$ \\
Coronary flow $(\mathrm{mL})$ & $7.6 \pm 1.4$ & $7.7 \pm 0.9$ & $9.5 \pm 1.9$ \\
Heart rate $(\mathrm{bpm})$ & $209 \pm 19$ & $167 \pm 14$ & $203 \pm 22$ \\
\hline
\end{tabular}

Data are reported as means $\pm S E$. There were no significant differences among groups $(P>0.05$, one-way ANOVA followed by Newman-Keuls post-test). CNTs: carbon nanotubes; HY-CNTs: carbon nanotubes associated with sodium hyaluronate; $\pm \mathrm{dT} / \mathrm{dt}$ : rates of contraction and relaxation.

their surface. Other factors that certainly contributed to the absence of harmful effects on cardiovascular function in the present study include the purity, physical properties, and structure of the biomaterial, in addition to the surface of the CNTs, experimental protocol, administration route, and concentration.

One issue that remains unanswered is the fate of CNTs after injection into tooth sockets. They might accumulate within macrophage phagosomes, nuclei, or in the reticuloendothelial system or extracellular matrix of the bone, or they may reach the blood stream. Although a number of studies have been conducted to elucidate the properties of the CNTs, pharmacological parameters, such as clearance, blood circulation, organ distribution, and accumulation remain to be determined (20). Therefore, the biodistribution and local inflammation caused by CNTs and HY-CNTs after injection into tooth sockets warrant further investigation.

It should be mentioned that only a few aspects of

\section{References}

1. Rugheimer L, Olerud J, Johnsson C, Takahashi T, Shimizu $\mathrm{K}$, Hansell P. Hyaluronan synthases and hyaluronidases in the kidney during changes in hydration status. Matrix Biol 2009; 28: 390-395, doi: 10.1016/j.matbio.2009.07.002.

2. Maha DR, Frederic T, Deschrevel B, Vincent JC, Bogdanowicz P, Boumediene K, et al. Differential effects of hyaluronan and its fragments on fibroblasts: Relation to wound healing. Wound Repair Regen 2008; 16: 274-287, doi: 10.1111/j.1524-475X.2007.00342.x.

3. Sasaki T, Watanabe C. Stimulation of osteoinduction in bone wound healing by high-molecular hyaluronic acid. Bone 1995; 16: 9-15, doi: 10.1016/8756-3282(95)80005-B.

4. Prince CW. Roles of hyaluronan in bone resorption. BMC Musculoskelet Disord 2004; 5: 12, doi: 10.1186/1471-24745-12.

5. Giavaresi G, Torricelli P, Fornasari PM, Giardino R, Barbucci R, Leone G. Blood vessel formation after softtissue implantation of hyaluronan-based hydrogel supplemented with copper ions. Biomaterials 2005; 26: 3001-3008, doi: 10.1016/j.biomaterials.2004.08.027.

6. Brown MB, Jones SA. Hyaluronic acid: a unique topical vehicle for the localized delivery of drugs to the skin. $J$ Eur cardiovascular function (blood pressure, HR, and cardiac function) were investigated in our present study. Thus, it is crucial to analyze other parameters, such as electrical properties of the heart, in order to establish the safety of CNTs under our experimental conditions. Also, the use of other concentrations of CNTs is required to confirm the low toxicity of these biocomposites.

In summary, our results, obtained 7 days following injection, indicated that the administration of low concentrations of CNTs or HY-CNTs directly into tooth sockets did not cause any significant changes in cardiovascular function in rats. The present findings support the possible use of these biocomposites in humans.

\section{Acknowledgments}

Research supported by FAPEMIG, CNPq, and CAPES.

Acad Dermatol Venereol 2005; 19: 308-318, doi: 10.1111/ j.1468-3083.2004.01180.x.

7. Mendes RM, Silva GA, Lima MF, Calliari MV, Almeida AP, Alves JB, et al. Sodium hyaluronate accelerates the healing process in tooth sockets of rats. Arch Oral Biol 2008; 53: 1155-1162, doi: 10.1016/j.archoralbio.2008.07.001.

8. Bhattacharyya S, Guillot S, Dabboue H, Tranchant JF, Salvetat JP. Carbon nanotubes as structural nanofibers for hyaluronic acid hydrogel scaffolds. Biomacromolecules 2008; 9: 505-509, doi: 10.1021/bm7009976.

9. Mendes RM, Silva GA, Caliari MV, Silva EE, Ladeira LO, Ferreira AJ. Effects of single wall carbon nanotubes and its functionalization with sodium hyaluronate on bone repair. Life Sci 2010; 87: 215-222, doi: 10.1016/j.lfs.2010.06.010.

10. Sa MA, Andrade VB, Mendes RM, Caliari MV, Ladeira LO, Silva EE, et al. Carbon nanotubes functionalized with sodium hyaluronate restore bone repair in diabetic rat sockets. Oral Dis 2013; 19: 484-493, doi: 10.1111/odi.12030.

11. Zhao B, Hu H, Mandal SK, Haddon RC. A bone mimic based on the self-assembly of hydroxyapatite on chemically functionalized single-walled carbon nanotubes. Chem Mater 2005; 17: 3235-3241, doi: 10.1021/cm0500399. 
12. Clichici S, Biris AR, Tabaran F, Filip A. Transient oxidative stress and inflammation after intraperitoneal administration of multiwalled carbon nanotubes functionalized with single strand DNA in rats. Toxicol Appl Pharmacol 2012; 259: 281 292, doi: 10.1016/j.taap.2012.01.004.

13. Helland A, Wick P, Koehler A, Schmid K, Som C. Reviewing the environmental and human health knowledge base of carbon nanotubes. Environ Health Perspect 2007; 115: 1125-1131, doi: 10.1289/ehp.9652.

14. Tong H, McGee JK, Saxena RK, Kodavanti UP, Devlin RB, Gilmour MI. Influence of acid functionalization on the cardiopulmonary toxicity of carbon nanotubes and carbon black particles in mice. Toxicol Appl Pharmacol 2009; 239: 224-232, doi: 10.1016/j.taap.2009.05.019.

15. Lanone S, Boczkowski J. Biomedical applications and potential health risks of nanomaterials: molecular mechanisms. Curr Mol Med 2006; 6: 651-663, doi: 10.2174/156652406778195026.

16. Dubin RA, Callegari G, Kohn J, Neimark A. Carbon nanotube fibers are compatible with Mammalian cells and neurons. IEEE Trans Nanobioscience 2008; 7: 11-14, doi:
10.1109/TNB.2008.2000144

17. Ladeira MS, Andrade VA, Gomes ER, Aguiar CJ, Moraes ER, Soares JS, et al. Highly efficient siRNA delivery system into human and murine cells using single-wall carbon nanotubes. Nanotechnology 2010; 21: 385101, doi: 10.1088/0957-4484/21/38/385101.

18. Kam NW, O'Connell M, Wisdom JA, Dai H. Carbon nanotubes as multifunctional biological transporters and near-infrared agents for selective cancer cell destruction. Proc Natl Acad Sci U S A 2005; 102: 11600-11605, doi: 10.1073/pnas.0502680102.

19. Hirsch A. Functionalization of single-walled carbon nanotubes. Angew Chem Int Ed Engl 2002; 41: 1853-1859, doi: 10.1002/1521-3773(20020603)41:11<1853::AIDANIE1853>3.0.CO;2-N.

20. Singh R, Pantarotto D, Lacerda L, Pastorin G, Klumpp C, Prato $\mathrm{M}$, et al. Tissue biodistribution and blood clearance rates of intravenously administered carbon nanotube radiotracers. Proc Natl Acad Sci U S A 2006; 103: 3357-3362, doi: 10.1073/pnas.0509009103. 